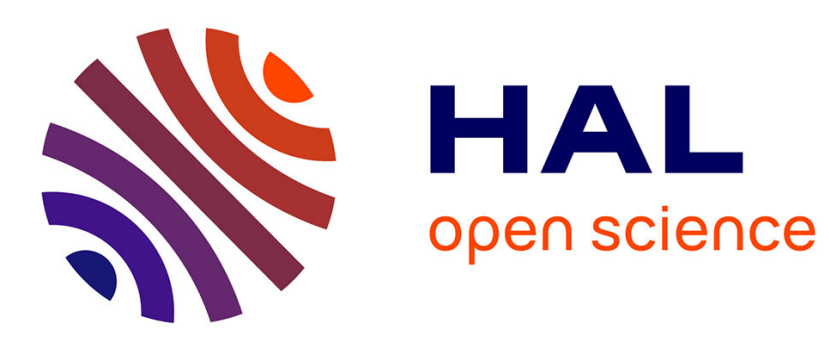

\title{
Revenue Sharing in Network Utility Maximization Problems
}

\author{
Isabel Amigo, Pablo Belzarena, Sandrine Vaton
}

\section{To cite this version:}

Isabel Amigo, Pablo Belzarena, Sandrine Vaton. Revenue Sharing in Network Utility Maximization Problems. Netnomics: Economic Research and Electronic, 2016, 17 (3), pp.255 - 284. 10.1007/s11066016-9112-4 . hal-01400498

\section{HAL Id: hal-01400498 \\ https://hal.science/hal-01400498}

Submitted on 22 Nov 2016

HAL is a multi-disciplinary open access archive for the deposit and dissemination of scientific research documents, whether they are published or not. The documents may come from teaching and research institutions in France or abroad, or from public or private research centers.
L'archive ouverte pluridisciplinaire HAL, est destinée au dépôt et à la diffusion de documents scientifiques de niveau recherche, publiés ou non, émanant des établissements d'enseignement et de recherche français ou étrangers, des laboratoires publics ou privés. 


\title{
Revenue Sharing in Network Utility Maximization Problems
}

\author{
Isabel Amigo • Pablo Belzarena • \\ Sandrine Vaton
}

\begin{abstract}
Alliances arise in a wide variety of domains, when a group of countries, political parties, people or other entities agree to work together because of shared interests or aims. They make sense, if the output obtained is somehow better than the outcome of acting individually. Revenue or cost sharing is key when determining if individuals are better off by contributing to an alliance or not. In our alliance each member owns a unique resource - or set of resources-, which is given to the alliance. The alliance sells services, which are supported thanks to one or a set of these resources. We focus on alliances that sell services in such a way that the total revenue of the alliance is maximized. We show that this kind of problems can be modeled through a Network Utility Maximization problem. We subsequently explore the problem of revenue sharing among the members of the alliance. Such a problem is a complex one since the interests of all participants must be ensured and correct incentives must be provided. We formally formulate the members' interests through a set of properties the revenue sharing method should verify. We then discuss the existing methods for revenue sharing and conclude that none of them verify the needed properties for the case of a revenue maximizing alliance. We finally propose a revenue sharing method based on projecting the contributions of each member of the alliance into an economic stable set. Through an exhaustive simulative study we conclude that our method provides, in addition to economic stabil-
\end{abstract}

\author{
I. Amigo, S.Vaton \\ Institut Mines Telecom, Télécom Bretagne \\ Technopole Brest-Iroise - CS 83818 \\ 29238 Brest Cedex 3, France \\ Phone: $(+33)(0) 229001111$ \\ E-mail: $\{$ isabel.amigo,sandrine.vaton\}@telecom-bretagne.eu \\ P. Belzarena \\ Facultad de Ingeniería, Universidad de la República \\ Julio Herrera y Reissig 565 - C.P. 11300 \\ Montevideo, Uruguay \\ Phone: (+598) 27110974 \\ E-mail: \{belza,iamigo\}@fing.edu.uy
}


ity, fairness among members and the right incentives to them. Through our analysis Network Service Provider alliances, which sell quality-assured data transport services, are considered as an application example.

Keywords Revenue sharing, Network utility maximization, Alliances, Cooperative game theory, Stability, Efficiency, Fairness, Monotonicity

\section{Introduction}

In different contexts where services must be provided throughout several geographical regions, with high availability and assured quality, collaboration arises as a viable way to reach the requirements of high-demanding clients without the need of ubiquitous deployment of redundant infrastructure. This statement is valid in a wide set of cases. The Internet connectivity provisioning context is one example, but it also happens, for instance, in the legacy telephony network, in cloud computing clusters, or even in railway companies and airlines. The collaboration ranges from merely network interconnection to common coordination and cooperation principles. The former occurs already in the Internet, the network of networks, for best-effort traffic, while examples of the latter are airline alliances.

After the introduction of Kelly's innovative network resource allocation method [10, Network Utility maximization (NUM) problems have been widely used in networking research activities (see e.g. 20 for a summary). In a NUM problem, network links capacities and a routing table determine linear constraints for the resource allocation problem. An optimization problem under such constraints is then established to maximize an aggregate objective function. This objective function is obtained as the sum of the utility of using each network's path. The decision variable is the amount of bandwidth to allocate to each network's path. Several proposals about applications further than networking can be found in the literature. In [17, the authors propose a real time pricing algorithm for smart grids based on the optimization of users' aggregated utility by selecting each user's energy consumption schedule, under constraints of energy availability. Yet another example can be found in [6], where a NUM problem is used for joint transmission scheduling and congestion control for adaptive streaming in the context of wireless Device-to-Device networks.

Synergy is one of the main attractions for the creation of an alliance, though it must be noticed that it is not achievable by the joint effort per se. Indeed, in order to provide higher revenues, coordination principles must be set in place, so as to allow for optimum resource allocation, along with the use of an appropriate revenue sharing mechanism, which guarantees that each member's share is at least equal to or greater than what he would obtain by acting alone.

Revenue sharing is also important for it acts as a mean to encourage the correct behavior of the alliance's members. Indeed, if, for instance, a member of the alliance that brings more clients to the alliance is compensated adequately, 
all members would dedicate efforts to attract clients, which in the end would result in higher revenues for the alliance.

This work is motivated by both the need of a fair and a right-incentive provider revenue sharing method, for revenue maximizing alliances. The NUM problem, which allows to maximize the alliance's revenue, makes the revenue sharing a challenge, since existing methods can not be applied directly and must be carefully revised. Moreover, the intersection of solutions that provide both incentive properties and fairness in the broad sense is not always clear. In this work we aim to find such intersection, while seeking as well implementable solutions.

Throughout this work we shall look at a Network Service Providers (NSP) alliance as an illustrative example. Such alliances have been proposed to offer quality-assured end-to-end data transport services between different parts of the globe 2. In addition to benefits such as, for instance, services' offer diversification, increase of the regions reached by those services, increase of the available resources and back-up paths, one of the main motivations for such alliances is the increase of the revenue of the NSPs that belong to it. This revenue increase comes from the fact that given a collaborative environment, services can be sold (or what is the same in this context, resources can be allocated) so as to maximize the revenue of the alliance as a whole, and moreover, possibly increase it with respect to what all its members would earn by acting by themselves. Besides, the advent of the software-defined networking (SDN) paradigm is likely to foster collaboration among different NSP, such as this kind of alliances. Indeed, in the SDN paradigm, network programmability and interoperability becomes possible, promising to simplify management.

In 4] we have introduced the problem under study and proposed guidelines for a method to solve it. The main contributions of this extended version of the work are: generalization of the problem, rich discussion of the desired properties, presentation and evaluation of existing methods which concludes that none of them are suitable for our problem and description of the solution proposal. The method is validated through exhaustive simulation studies.

The remainder of this paper is organized as follows. In section 2 we introduce notation and formally define the problem. We then discuss the desired properties for the revenue sharing mechanism in Section 3. In Section 4 we review the most common sharing rules used in the economics field, and we argue on why they are not useful when the revenue is determined through a NUM problem. In Section 5 we present a new method, which provides a solution that guarantees stability and efficiency in economic terms. In Section 6we present our simulation studies. In particular, results demonstrate the correct behavior of the proposed method towards desired properties, such as fairness and monotonicity. Finally, in Section 7 we discuss some implementation considerations and conclude in Section 8 . 


\section{Mathematical Model}

We now introduce some notations so as to formally represent the working scenario.

\subsection{The Alliance}

The alliance is formed by a set $N$ of members. Each member of the alliance is identified by $n$, where $n=1 \ldots|N|$, and provides to the alliance a quantity $c_{n}$ of a resource. We shall refer to vector $c$ defined as $c=\left\{c_{n}\right\}_{n \in N}$ as the stock vector. The alliance sells different services, the services belong to the set $S$ and are identified by $s$, where $s=1 \ldots|S|$. Each service $s$ needs a given combination of resources, and a given quantity of each resource. We represent these combinations with the $|N| \times|S|$ matrix $R$, where $|\bullet|$ stands for the cardinality of $\bullet$. Then, entry $R_{n, s}$ means that service $s$ uses an amount $R_{n, s}$ of the resource provided by $n$. The amount of a given service $s$ to deliver, or sell, or produce by the alliance, is denoted by $a_{s}$. Vector $a$ is defined as $a=\left\{a_{s}\right\}_{s \in S}$. Each service $s$ has a utility function associated to it, referred to as $U_{s}\left(a_{s}\right)$. We assume that $U_{s}\left(a_{s}\right)$ is known and, as usual in this context, it is a strictly concave function of the amount of services sold. This utility is interpreted as the revenue each service can provide to the alliance, in some unit, as a function of the amount of service sold. In addition, $a_{s}$ are assumed to take continuous values.

Example Consider an alliance of NSPs.The interest of the collaboration is to provide end-to-end quality-assured connectivity services. Each NSP member of the alliance can be abstracted to a node. Each NSP or node $n \in N$ offers connectivity to neighboring nodes with a certain capacity, $c_{n}$, which is expressed in any data transfer unit. The services offered by the alliance, the end-to-end connectivity services, are described by routing matrix $R$ whose binary entries $R_{n, s}$ are equal to 1 if service $s$ 's route goes through $N S P n$, and 0 otherwise. The amount of bandwidth dedicated to each service is then noted by $a_{s}$. Utilities represent the price paid for each service and are functions of the amount of bandwidth of a service. For more detail in the NSP alliance model, the reader is referred to 3 .

Please note that $c$ can be interpreted as a resource contribution, however, it can not be interpreted as a monetary contribution, since a contribution in resources does not necessarily imply increasing the alliance's revenue. Next subsection introduces how resources are allocated, and thus, in which case they are translated into a monetary contribution.

\subsection{Resource Allocation}

We work with a revenue maximizing alliance, meaning that the resources provided by the members are allocated to services in such a way that the revenue 
of the alliance is maximized. This can be formalized following the approach proposed in the seminal work of Kelly [10. In such work, a NUM problem is proposed to solve the resource allocation in such a way that a global network utility function is maximized while link capacities are respected. In our case we apply the same principle to our model of an abstracted alliance. Indeed, in our scenario the amount of resources allocated to each type of service must be such that the revenue perceived by the alliance is maximized, while the resource constraints are fulfilled.

We shall refer to the set of members $N$ as the grand coalition and subgroups of members shall receive the name of sub-coalitions or sub-alliances. For convenience, as we shall shortly see, we shall define the resource allocation problem for the grand coalition as well as for any sub-coalition. Let $\mathcal{Q} \subseteq N$ be any sub-coalition of $N$ and let $c^{\mathcal{Q}}$ be the stock vector restricted to sub-coalition $\mathcal{Q}$, that is:

$$
c_{n}^{\mathcal{Q}}=c_{n} \text { if } n \in \mathcal{Q} ; c_{n}^{\mathcal{Q}}=0 \text { otherwise. }
$$

Then, the resource allocation problem is formalized in the following optimization problem:

\section{Problem 1}

$$
\begin{aligned}
& \max _{a} \sum_{s \in S} U_{s}\left(a_{s}\right) \\
& \text { s.t. } R a \leq c^{\mathcal{Q}}
\end{aligned}
$$

In Problem 1 we have not considered the fact that the alliance may not want to sell a given service if the incomes perceived by doing so are lower than a certain bound. However, we can model this situation by defining a cost function of the allocated services $\kappa_{s}\left(a_{s}\right)$ for each service $s \in S$ and redefine Problem 1 considering as objective function $\sum_{s \in S}\left[U_{s}\left(a_{s}\right)-\kappa_{s}\left(a_{s}\right)\right]$. Provided the cost function is convex, this will result in a problem analogous to Problem 1. For the sake of notations simplicity we shall not consider the cost function hereafter.

Another interesting remark can be made considering costs and resources. Is it convenient for a member to increase its resources? This can not be answered without considering a revenue sharing mechanism, and in particular, the properties of the revenue sharing mechanism. This aspect will thus be addressed in Section 3 ,

\subsection{Revenue Sharing}

Revenue sharing problems constitute one of the objects of study of Game Theory. Indeed, a revenue -or cost- sharing problem involves the interaction of several agents or actors which may compete or collaborate acting in a rational way. We shall thus from now on adopt the Game Theory's nomenclature and refer to the members of the alliance also as players. 


\begin{tabular}{|c|c|}
\hline Variable & Meaning \\
\hline $\mathcal{Q} \subseteq N$ & Grand coalition, set of all members in the alliance. \\
$S$ & Sub-coalition. \\
$R=\left\{R_{n, s}\right\}_{n \in N, s \in S}$ & Set of available services offered by the alliance. \\
$a=\left\{a_{s}\right\}_{s \in S}$ & Service $s$ uses an amount $R_{n, s}$ of $n$ 's resource. \\
$U=\left\{U_{s}\left(a_{s}\right)\right\}_{s \in S}$ & Vector, amount sold of each service $s \in S$. \\
$c=\left\{c_{n}\right\}_{n \in N}$ & Utility functions of each service. \\
$c^{Q} \cdot c_{n}^{\mathcal{Q}}=c_{n}, \forall n \in \mathcal{Q}, c_{n}^{\mathcal{Q}}=0 \forall n \notin \mathcal{Q}$ & Stock vector, $c_{n}$ amount of $n$ 's resource. \\
$V(\mathcal{Q})=\max _{a} e^{T} \cdot U(a), s \cdot t \cdot R a \leq c$ & Stock vector for sub coalition $\mathcal{Q}$. \\
$x_{n}=\Phi_{n}(N, c, U)$ & Revenue of the coalition $\mathcal{Q} \subseteq N$. \\
$x=\left\{x_{n}\right\}_{n \in N}=\Phi(c, N, U)$ & Revenue share of member $n$. \\
$v_{n}=V(N)-V(N \backslash\{n\})$ & Revenue share vector. \\
\hline
\end{tabular}

Table 1: Summary of notations used throughout the paper

Our problem is to find a suitable method to share the revenue of the coalition among its members. Let us first introduce the notion of revenue and of revenue share, and the notations we shall use to represent them. We define the revenue function $V$ by the solution of Problem [1] which states that resources are allocated in such a way that the revenue of the alliance is maximized, while respecting the resource constraints.

The revenue function associates to each sub-coalition $\mathcal{Q} \subseteq N$ with resources $c^{\mathcal{Q}}$ and utility functions $U=\left\{U_{s}\right\}_{s \in S}$, a real value $V\left(\mathcal{Q}, c^{\mathcal{Q}}, U\right)$. We also accept the notation $V(\mathcal{Q})$ to indicate the total revenue of coalition $\mathcal{Q} \subseteq N$, where resources and utility functions are implicit or $V(\mathcal{Q}, c)$ when utility functions are implicit by context.

The total revenue is shared among all members in $N$ according to the sharing function $\Phi(N, c, U)$ which computes a revenue sharing vector $\left\{\Phi_{n}\right\}_{n \in N}$, where $\Phi_{n}$ is the total revenue that player $n$ will receive, expressed in some monetary unit. This function depends on the coalition $N$, the resources $c$ and the utility functions $U$. For convenience and brevity, we shall also use the shorter notation $x$ to denote the revenue sharing vector, where $x \in \mathbb{R}^{|N|}$ is a column vector containing on each component $x_{n}, n \in N$, the revenue share of member $n$, when the values of $N, c$ and $U$ are implicit by context.

The notations presented in this section, along with further notations introduced later in this paper, are summarized in Table 1 .

\section{Desired Properties of the Revenue Sharing Mechanism}

Two main objectives motivate the desired properties of a revenue sharing method. First, to provide with fair allocations. Second, to provide to the members the right incentives to remain in the alliance and to contribute to it. The properties discussed below, are usually discussed in cost/revenue sharing problems, with slightly different definitions (see for instance [7, 9, [11]). We select from the literature the ones that we believe are more relevant to our problem, argument on why they are relevant and formally define them. 


\subsection{Efficiency and Stability}

The mechanism should distribute all the alliance's revenue among its members, that is what we call efficiency, which is defined as follows.

Efficiency. $\Phi(N, c, U)=x$ is efficient if

$$
\sum_{n: n \in N} x_{n}=V(N)
$$

In addition, in order to assure the sustainability of the alliance, the mechanism should not provide incentives to any sub group of members to break up the grand coalition. That is, no sub-coalition should have economic incentives to form a smaller coalition outside the alliance, since this would lead to instabilities in the alliance. This is the so-called stability property.

Stability. $\Phi(N, c, U)=x$ is stable if

$$
\sum_{n: n \in \mathcal{Q}} x_{n} \geq V(\mathcal{Q}), \forall \mathcal{Q} \subseteq N
$$

The stability definition requires the shares to be such that, for every possible sub-coalition, the sum of the shares of the members belonging to that sub-coalition are at least as large as the revenue that sub-coalition would perceive. This property is also usually referred as the stand alone property. Please note that this definition also implies that the revenue perceived by each member $n \in N$ in the coalition is not less than the revenue it could achieve alone, i.e. $x_{n} \geq V(\{n\}), \forall n \in N$. The set of points that verify Inequalities (3) constitutes the so-called core set in the context of Coalitional Game Theory. The reader is referred to [19] for more details on the core concept and on coalitional game theory.

\subsection{Monotonicity}

The mechanism should provide the right incentives to the members to perform actions towards an increase in the alliance's revenue. Incentives, in our context, means having economic reasons to perform some action. In particular, the economic reasons a player has for performing a given action are modeled by the increase that this action can provide on his or her own revenue. We formalize the concept of incentives through the monotonicity property. Several flavors of monotonicity properties can be found in the literature. We now discuss three of the more relevant ones to our problem. We finally keep one definition of monotonicity as desirable for our revenue sharing mechanism.

$N$-resource-monotonicity. $\Phi(N, c, U)$ is N-resource-monotonic if given two vectors of resources $c$ and $\hat{c}$, such that $\hat{c}_{k} \geq c_{k}$ and $\hat{c}_{n}=c_{n} \forall n \neq k$ then $\Phi_{n}(N, \hat{c}, U) \geq \Phi_{n}(N, c, U) \forall n \in N$.

$\mathrm{N}$-resource monotonicity provides incentives to each member to increase their amount of resource or not to decrease it, while members not increasing their resources have no incentive to discourage such increase, which makes it a 
very compelling property. However, it is a very strong requirement. Moreover, it can be proved that in the general case of games with a feasible, efficient, and stable solution set, there is no way to split the revenue that provides with jointly efficiency, stability and $N$-resource-monotonicity [21] 9 .

As a consequence of the previously cited impossibility result, we shall admit a less restrictive property, which we simply call resource-monotonicity, and only ask for a non-decrease on the revenue of that member increasing its stock. More formally:

Resource-Monotonicity. Given two vectors of resources $c$ and $\hat{c}$, such that $\hat{c}_{n}=c_{n} \forall n \in N \backslash\{k\}$ and $\hat{c}_{k} \geq c_{k}, \Phi$ is resource-monotonic if $\Phi_{k}(N, \hat{c}, U) \geq$ $\Phi_{k}(N, c, U)$.

The resource-monotonicity property means that if a member increases its amount of resources then its revenue will as well increase or remain the same. This property is usually referred to as resource incentive, or monotonicity in the resources, in the context of coalitional game theory.

Another interesting property related to monotonicity is the one that evaluates the influence of new members entering the alliance, usually referred as to population-monotonicity.

Population-monotonicity. Given stock vector $c$, a revenue sharing rule $\Phi$ is population-monotonic if $\forall S, T \subseteq N$ such that $S \subset T, \Phi_{n}\left(T, c^{T}, U\right) \geq$ $\Phi_{n}\left(S, c^{S}, U\right) \forall n \in S$.

A population-monotonic revenue sharing rule guarantees that the entrance of a new member to the alliance does not reduce the revenue of each of the members already there. However, we focus on the study of fixed alliances, and not on the dynamics of how to build them. The alliance could be set in place for reasons further to the ones captured by the revenue function, such as business agreements and geographical coverage. Thus, we shall not consider this property as mandatory for a sharing rule. It is rather a property that should be checked whether it is verified or not given a particular alliance.

We shall herein seek for a revenue sharing method satisfying resourcemonotonicity, and refer to this property simply as monotonicity. Regarding incentives, the monotonicity property still provides to a member incentives to increase its resources towards the alliance. In the context of coalitional game theory, $N$-resource-monotonicity is usually asked, so as to guarantee that no player would have incentives to block some other player's action towards the increase of the revenue of the whole alliance. In particular, if we ask simply for monotonicity and not for $N$-resource-monotonicity, it could happen that some members in the alliance have no interest in the resource increase of other members. However, even if the resource increase of one member could decrease some other member's revenue, we claim that this situation could act itself as an incentive to members to remain competitive in terms of their resource contribution towards the alliance. 


\begin{tabular}{|c|c|}
\hline Property name & Property meaning \\
\hline Stability & $\sum_{n: n \in \mathcal{Q}} x_{n} \geq V(\mathcal{Q}), \forall \mathcal{Q} \subseteq N$ \\
Efficiency & $\sum_{n: n \in N} x_{n}=V(N)$ \\
& Monotonicity \\
Eq. treat. of eq. & If $\hat{c}_{n}=c_{n} \forall n \in N \begin{array}{l}\{k\} \text { and } \hat{c}_{k} \geq c_{k}, \text { then } \Phi_{k}(N, \hat{c}, U) \geq \Phi_{k}(N, c, U) . \\
\text { Order preserving }\end{array}$ if $v_{n}=v_{j}$ then $x_{n}=x_{j}$ \\
No free riders & If $v_{n} \geq v_{j}$ then $x_{n} \geq x_{j}$ \\
if $v_{n}=0$ then $x_{n}=0$ \\
\hline
\end{tabular}

Table 2: Summary of the desirable properties

\subsection{Fairness}

There is not a general consensus in the literature regarding the notion of fairness. Moreover, the properties enumerated before can as well be interpreted as fairness. Indeed, the stability property states that every sub-coalition will get an aggregate share of at least the sub-coalition's revenue. The monotonicity property, besides providing incentives to increase resources, can as well be interpreted as a fairness one. Indeed, if one member makes an effort to improve its stock, then it deserves to be rewarded adequately.

In addition to the properties stated so far, we propose some common in the literature and intuitive rules that should be fulfilled in order to be fair. We base our definitions on the contribution of each member, defined for member $n$ as $v_{n}=V(N)-V(N \backslash\{n\})$. The so-called order preserving property is then defined as follows: If $v_{n} \geq v_{j}$ then $x_{n} \geq x_{j}$. If the previous inequalities are interpreted as strict, that is if $v_{n}=v_{j}$ implies $x_{n}=x_{j}$, this has received the name of equal treatment of equals. In addition we propose that if $v_{n}=0$ then $x_{n}=0$, which we shall call no free-riders and can be seen as a particular case of the so-called dummy property in the context of coalitional game theory. All in all, we define fairness as follows.

Fairness. $\Phi$ is fair if it is order preserving, guarantees equal treatment of equals and no free-riders.

The choice of the contribution vector to evaluate fairness is important. Some classical rules of revenue or cost sharing propose fairness criteria based on the resources or costs of each agent. However, in our case, we base the fair principle in the contribution of each member in terms of revenue rather than in terms of resources, since what is important in the alliance is not only the resources provided by each member but as well how much value we can get from the resources. Vector $v$ is a measure that takes into account both relevant components, namely the resource itself and its value.

For the reasons exposed in this section, we shall seek for a revenue sharing method which fulfills stability, efficiency, fairness and monotonicity. The meaning of these properties is summarized in Table 2. 


\section{State-of-the-Art Sharing Methods}

We now present existing revenue sharing techniques, which have been proposed in the field of economics and some of them have been widely used for solving revenue sharing problems. A detailed review of costs and revenue sharing rules can be found in [9] and a discussion of bargaining methods in [16. In particular, we discuss the properties they verify, and their behavior with respect to our desirable properties, for concluding that none of them verifies them all.

\subsection{The Shapley Value}

The Shapley value, proposed by Lloyd Shapley in 1953 [18, is probably the most well known technique to perform revenue sharing in an association or coalition. It has been widely used in the literature for its good properties, which we shall review in the following. The Shapley value provides a closedform expression to compute the share of each agent in a cooperative context. Intuitively, it can be interpreted as computing the average contribution of each agent to the coalition.

With our notations, the Shapley value for player $n \in N$ is defined as:

$$
x_{n}^{s h}=\frac{1}{|N| !} \sum_{\mathcal{Q} \subseteq N \backslash\{n\}}|\mathcal{Q}| !(|N|-|\mathcal{Q}|-1) ![V(\mathcal{Q} \cup\{n\})-V(\mathcal{Q})] .
$$

Example Consider again an NSP alliance. Consider Topology A in Fig. 1a, capacities are equal to 1 unit for all nodes. Consider as well that utility functions $U=\left(u_{1}, u_{2}\right)$ are as specified in Table 3a, The solution to Problem 11 leads to the following revenues: $V(N)=V(\{2,3\})=10, V(\{1,3\})=5$.

The Shapley value for node 1 is given by:

$$
\begin{aligned}
x_{1}^{s h} & =\frac{1 ! 1 !}{3 !}[V(\{1,2\})-V(\{2\})]+\frac{1 ! 1 !}{3 !}[V(\{1,3\})-V(\{3\})] \\
& +\frac{2 ! 0 !}{3 !}[V(N)-V(\{2,3\})] \\
& =\frac{1}{6} 0+\frac{1}{6} 5+\frac{1}{3} 0=\frac{5}{6} .
\end{aligned}
$$

Analogously, for node 2 we obtain $x_{2}^{s h}=\frac{10}{3}$, and for node $3 x_{3}^{s h}=\frac{35}{6}$

The following three properties completely characterize the Shapley value, as proved by Shapley [18.

- Dummy player: if $n$ is a dummy player (i.e. a player whose contribution to the coalition is the same as the one he would achieve on his own) then $x_{n}=V(\{n\})$.

- Symmetry: if $n$ and $j$ contribute the same to any coalition then $x_{n}=x_{j}$.

- Additivity: let $U^{(1)}$ and $U^{(2)}$ be two different utility functions, if $\Phi_{n}\left(N, c, U^{(1)}+U^{(2)}\right)=$ $\Phi_{n}\left(N, c, U^{(1)}\right)+\Phi_{n}\left(N, c, U^{(2)}\right) \forall n \in N$. 


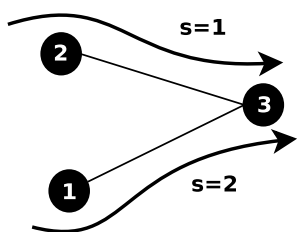

(a) Topology A. The feasible set can be a region under such a topology.

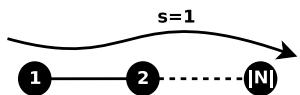

(b) Topology B. The Friedman-Moulin rule provides the wrong incentives under such a topology.

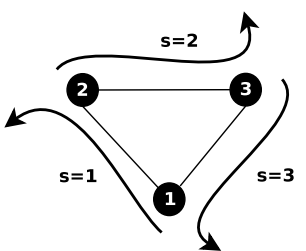

(c) Topology C. The feasible set can be empty under such a topology.

Fig. 1: Example: NSP alliances topologies

In addition, it is efficient (it shares the total revenue), resource-monotonic and has balanced contributions [12].

A sharing rule has balanced contributions if and only if for any two players $i, j \in N, i$ 's contribution to $j$ is equal to $j$ 's contribution to $i$, that is $\Phi_{i}(\mathcal{Q}, c, U)-\Phi_{i}(\mathcal{Q} \backslash\{j\}, c, U)=\Phi_{j}(\mathcal{Q}, c, U)-\Phi_{j}(\mathcal{Q} \backslash\{i\}, c, U) \forall \mathcal{Q} \in N$ : $|\mathcal{Q}| \geq 2$.

Example Recalling the previous example, we can readily verify that the solution obtained is efficient, that is $x_{1}^{s h}+x_{2}^{s h}+x_{3}^{s h}=\frac{5+20+35}{6}=10=V(N)$. We can as well verify that the solution has balanced contributions according to the previous definition. Take for instance nodes 1 and 2, and let $\Phi_{n}^{s h}(N, c, U)$ be $n$ 's Shapley value for coalition $N$, stock vector $c$ and utility function $U$. The revenues of the different sub-coalitions for this example are shown in Table 3b, Then, $\Phi_{1}^{s h}(N, c, U)-\Phi_{1}^{s h}(N \backslash\{2\}, c, U)=\frac{5}{6}-\frac{5}{2}=\frac{-10}{6}$ and $\Phi_{2}^{s h}(N, c, U)-\Phi_{2}^{s h}(N \backslash\{1\}, c, U)=\frac{10}{3}-\frac{10}{2}=\frac{-10}{6}$. And analogously for the other players and sub-coalitions $\{1,2\},\{2,3\},\{1,3\}$.

The following theorem states that the Shapley value for revenue maximizing alliances verifies resource-monotonicity.

Theorem 1 Monotonicity of the Shapley value. Let $(N, V, c)$ be a coalitional game where the set $N$ of members of the alliance are the players, c represents the stock of resources for each and $V$ is the revenue function defined by Problem 1. If $n \in N$ increases its stock then its revenue share (i.e. $\Phi_{n}^{s h}$ ) will not be decreased. That is, letting $\hat{c}$ represent the stock vector where only $n$ 's stock is increased, $\Phi_{n}^{s h}(N, \hat{c}, U) \geq \Phi_{n}^{s h}(N, c, U)$, where $\Phi_{n}^{s h}(N, c, U)$ is the Shapley value of $n$ given the coalitional game $(N, V, c)$.

Proof By definition of Shapley value $\Phi_{n}^{s h}(N, \hat{c}, U)=\frac{1}{|N| !} \sum_{\mathcal{Q} \subseteq N \backslash\{n\}}|\mathcal{Q}| !(|N|-$ $|\mathcal{Q}|-1) ![V(\mathcal{Q} \cup\{n\}, \hat{c})-V(\mathcal{Q}, \hat{c})]$, where $V(\mathcal{Q}, \hat{c})$ as defined above represents the worth function for sub-coalition $\mathcal{Q}$ when the stock is given by vector $\hat{c}$. 


$$
\Phi_{n}^{s h}(N, \hat{c}, U)=\frac{1}{|N| !} \sum_{\mathcal{Q} \subseteq N \backslash\{n\}}|\mathcal{Q}| !(|N|-|\mathcal{Q}|-1) ![V(\mathcal{Q} \cup\{n\}, \hat{c})-V(\mathcal{Q}, c)],
$$

holds since the revenue function of any coalition without member $n$ is the same, regardless the stock $n$ has.

By subtracting $n$ 's revenue share with and without increasing its stock we have that $\Phi_{n}^{s h}(N, \hat{c}, U)-\Phi_{n}^{s h}(N, c, U)=\frac{1}{|N| !} \sum_{\mathcal{Q} \subseteq N \backslash\{n\}}|\mathcal{Q}| !(|N|-|\mathcal{Q}|-1) ![V(\mathcal{Q} \cup$ $\{n\}, \hat{c})-V(\mathcal{Q} \cup\{n\}, c)]$.

We now determine if the inequality $V(\mathcal{Q} \cup\{n\}, \hat{c}) \geq V(\mathcal{Q} \cup\{n\}, c) \forall \mathcal{Q} \subseteq N \backslash$ $\{n\}$ holds. Indeed, $V$ is the solution to Problem 1, which is the maximization of a concave function with convex constraints. By increasing $n$ 's stock we relax such problem, thus doing so yields to greater or equal solutions, which concludes the proof.

In spite of fulfilling the aforementioned compelling properties, the Shapley value is not suitable for our problem, as it does not always provide stable solutions. That is to say, shares computed through the Shapley value do not always fulfill (3). Nonetheless, its great popularity in previous work is due to the fact that it is proven that for a special case of games, it provides with stable solutions (see e.g. [19]). This special case of games are convex games, which occur in many cases. A cooperative game with set of players $N$ and revenue function $V$ is convex if for any two sub-coalitions $W, Y \subseteq N, V(W)+$ $V(Y) \leq V(W \cap Y)+V(W \cup Y)$. Moreover, for convex games, the Shapley value provides with stable, efficient and $N$-monotonic solutions (see e.g. [9]).

As for our problem, the cooperative game defined by the revenue maximizer alliance with players $N$, stock vector $c$ and utility functions $U$ can be nonconvex. Solutions through Shapley value can thus lie outside the core, as shown in the following example.

Example Consider again the previous example, given by topology A in Fig. 1a, nodes with 1 unit of capacity, and utility functions given by Table 3a Consider coalition $N$ and sub-coalitions $W=\{1,3\}$ and $Y=\{2,3\}$, whose revenues are determined by solving Problem 1 and are given in Table $3 \mathrm{~b}$. We can readily check that $V(W \cap Y)+V(W \cup Y)=0+V(N)=10$, which is smaller than $V(W)+V(Y)=5+10=15$. Hence, the game defined by $N$ and $V$ is not a convex one.

In order to be stable, the Shapley value should verify Inequalities (3). In particular, in this example we have, $x_{2}^{s h}+x_{3}^{s h}=\frac{20}{6}+\frac{35}{6}=\frac{55}{6}<10=V(\{2,3\})$. Hence, stability inequalities are not satisfied.

This example also shows that the Shapley value does not fulfill the no free riders property. Indeed, node 2's contribution to the coalition, that is $v_{2}=V(N)-V(N \backslash\{2\})=0$, while $x_{2}^{s h} \neq 0$. 


\begin{tabular}{c|c|c}
\multicolumn{3}{c}{$U_{s}(s)=\alpha_{s} \cdot \log \left(1+\beta_{s} \cdot a_{s}\right)$} \\
\hline Service & $\alpha_{s}$ & $\beta_{s}$ \\
\hline $\mathrm{s}=1$ & 14.427 & 1 \\
$\mathrm{~s}=2$ & 7.2135 & 1 \\
\hline
\end{tabular}

(a) Utility functions

\begin{tabular}{c|c|c}
\hline $\mathcal{Q}$ & $a^{*}$ & $V(\mathcal{Q})$ \\
\hline$N=\{1,2,3\}$ & $(0,1)$ & 10 \\
$W=\{1,3\}$ & $(0,1)$ & 10 \\
$Y=\{2,3\}$ & $(1,0)$ & 5 \\
\hline
\end{tabular}

(b) Revenue of the grand coalition and two sub-coalitions

Table 3: Numerical values for topology A

\subsection{The Proportional Share}

One of the simplest ways to perform the revenue sharing is to split revenues proportionally to some contribution measurement. In our case, as explained above, vector $v=\left\{v_{n}\right\}_{n \in N}$ where, we recall, $v_{n}=V(N)-V(N \backslash\{n\})$, quantifies this contribution. The proportional share is then defined as:

$$
x_{n}^{p r}=\frac{v_{n}}{\sum_{n^{\prime} \in N} v_{n^{\prime}}} V(N)
$$

Example Consider again a NSP alliance whose topology is given by Topology A in Fig. 1a and that the utility functions, and thus revenues, are the same as before and are shown in Table 3. The proportional share for node 1 gives:

$$
\begin{aligned}
x_{1}^{p r} & =\frac{V(N)-V(\{2,3\})}{\sum_{n \in N} V(N)-V(N \backslash\{n\})} V(N) \\
& =\frac{10-5}{(10-5)+(10-10)+(10-0)} 10=\frac{10}{3} .
\end{aligned}
$$

Analogously we obtain, $x_{2}^{p r}=0$ and $x_{3}^{p r}=\frac{20}{3}$.

The proportional share a priori seems to be a very attractive distribution rule. It fulfills the properties of efficiency and fairness (see e.g. [9]) and it is very simple to compute. However, stability is not guaranteed by this sharing rule, as we shall show later on in Section 6 .

\subsection{The Aumann-Shapley Rule}

The Aumann-Shapley Rule for cost sharing [5] was introduced by Shapley and Aumann in 1974, and can be applied analogously for a revenue sharing problem. The idea of this rule is to compute the revenue share of a member $n \in N$ as its average marginal revenue along a certain path going from a stock equal to 0 to $c_{n}$. More precisely, the share for $n \in N$ according to this rule is defined as:

$$
x_{n}^{a s}=\int_{0}^{c_{n}} \partial_{n} V\left(N, \frac{t}{c_{n}} c\right) d t=c_{n} \int_{0}^{1} \partial_{n} V(N, t c) d t,
$$

where the notation $\partial_{n} V(N, c)$ means the first order derivative of $V$ at $c$ with respect to $c_{n}$. Please note that in Equation (6) we have used the alternative 
notation for $V$ where its dependency on the sub-coalition and the available resources are explicitly mentioned.

In our problem, the derivative of $V$ with respect to $c_{n}$ is not defined for all values of $c_{n}$. In addition, this rule applied to our problem can provide with the wrong incentives, as illustrated by the following example.

Example Consider a NSP alliance. Consider Topology B given by Fig. 1b and that the capacity of all nodes but node 1 are equal to a value $c_{\max }$, and node 1 's capacity is equal to $c_{\min }$, with $c_{\min }<c_{\max }$. The revenue of the alliance, we recall, is determined by Problem 1 . Consider that utility function $U_{1}\left(a_{1}\right)$ 's derivative with respect to $c_{1}$ is $u_{1}\left(a_{1}\right)$. In this case the Aumann-Shapley rule gives to node 1 :

$$
\begin{aligned}
x_{1}^{a s} & =\int_{0}^{c_{\min }} \partial_{1} V\left(N,\left(t, \frac{t \cdot c_{\max }}{c_{\min }} \ldots, \frac{t \cdot c_{\max }}{c_{\min }}\right)\right) d t \\
& =\int_{0}^{c_{\min }} u_{1}(t) d t=U_{1}\left(c_{\min }\right)-U_{1}(0) .
\end{aligned}
$$

Analogously, we can compute the share for every node $n \in\{2 \ldots|N|\}$. Since $V(N, t c)$, which is the solution to Problem 1 is always determined by the first component of vector $c$ (the smallest component) its derivative with respect to $c_{n}$ is equal to zero $\forall n \in\{2 \ldots|N|\}$. Hence, $x_{n}^{a s}=0$.

In particular, this example violates resource monotonicity since we can find a vector $c$ and a vector $\hat{c}$ such that $\hat{c}_{n}=c_{n} \forall n \in N \backslash\{k\}$ and $\hat{c}_{k} \geq c_{k}$ and such that $\Phi_{k}(N, \hat{c}, U)<\Phi_{k}(N, c, U)$. Indeed, let $\hat{c}_{n}=c_{n}=c_{\max } \forall n \in\{2 \ldots|N|\}$. Let $c_{1}=c_{\min }<c_{\max }$ and $\hat{c_{1}}>c_{\max }$ then $\Phi_{k}(N, \hat{c}, U)<\Phi_{k}(N, c, U)$.

From this simple example we can readily see that this rule applied to our scenario can provide the wrong incentives. Indeed, every node except node 1 is interested in decreasing its capacity so as to become the bottleneck, as this would give that node a non-null share. Notice that in order to be efficient, $U_{1}(0)$ should be equal to zero, which is likely to occur.

\subsection{The Friedman-Moulin Rule}

This rule was proposed by Friedman and Moulin in 1999 8. We introduce the operator $\wedge$, which is defined for two vectors $r$ and $q \in \mathbb{R}^{|N|}$ as $q \wedge r=$ $\min \left(q_{n}, r_{n}\right) n \in N$ and column vector $e$, which is of dimension $|N|$ and has all its components equal to one. This rule is similar to the Aumann-Shapley one, in terms that it integrates marginal revenues, but in this case the integration is done through a different path. According to the Friedman-Moulin rule, the share for member $n \in N$ is calculated as:

$$
x_{n}^{f m}=\int_{0}^{c_{n}} \partial_{n} V(N,(t \cdot e) \wedge c) d t .
$$

This rule can not be applied in our context since $V$ might not be derivable along the whole path, as seen in the following example. 
Example Consider again Topology B given by Fig. 1b, where the capacities of all nodes but node 1 are equal to a value $c_{\max }$, and node 1 's capacity is equal to $c_{\min }$, with $c_{\min }<c_{\max }$, as in the previous example. The Friedman-Moulin rule applied to node 1 is as follows:

$$
x_{1}^{f m}=\int_{0}^{c_{\text {min }}} \partial_{1} V(N, t \cdot e) d t,
$$

which is not defined along the whole path, since $\partial_{1} V\left(N, t^{-} \cdot e\right)=u_{1}(t)$ and $\partial_{1} V\left(N, t^{+} \cdot e\right)=0$

\subsection{The Nash Bargaining Solution}

Bargaining processes model classical economic problems in which players negotiate in order to collectively choose an outcome, in situations where there is no consensus about which the best outcome is (see for instance [14). Formalizing the output of this problem is not an easy task, since there is a plethora of actions that a player could take throughout the process, and a wide variety of ways to carry out the negotiation, involving different sequential order on which the players play and time-frames on which they take decisions or the game ends. In particular, according to the bargaining power, preferences and impatience of the different players, different outputs could be obtained. Bargaining situations lie in the core of the interests of game theory, discipline which has provided different formalizations of such problems, among which the solution concepts derived first by Nash in 1950 [13] and then by Rubinstein in 198215 are seminal ones.

We shall focus on the Nash Bargaining solution. Following Nash's terms, bargaining describes a situation where: a mutual agreement can be decided upon, a conflict of interest exists about which agreement to adopt, and the agreement needs the approval of all involved actors. In our revenue sharing problem, alliance members interact in order to decide on how to split the revenue that they achieve by the joint work.

In order to apply the Nash bargaining solution to our context, we need to define the -compact and convex- set of all possible outcomes, model each member's bargaining power (weight for negotiating), their disagreement point (outcome when there is no agreement), and define each player's utility function, i.e. their preferences over the set of possible outcomes. The Nash bargaining solution needs that there exits within the set of possible outcomes an outcome $x$ such that $x_{n}>d_{n}$, for all $n \in N$, where $d_{n}$ is $n$ 's disagreement point.

In this work we assume that all players have linear utility functions, and that they value the revenue shared obtained in the same way. We shall leave out of scope the modeling of each player's utility functions.

With respect to bargaining power, we propose two alternative models. One is to consider the members' contribution to the alliance as the bargaining power. That is to say, $\alpha_{n}=v_{n}$, for all $n \in N$. Alternatively, one can consider 
a member's stock of resource as its bargaining power. That is to say, $\alpha_{n}=c_{n}$, for all $n \in N$.

The disagreement point can be intuitively modeled by the stand alone revenue of each player, i.e. $d_{n}=V(\{n\})$, for all $n \in N$. The total amount to share in our context is $V(N)$, which bounds the set of possible outcomes.

We define an economically sensible alliance as an alliance whose revenue is larger than the sum of all revenues each member would perceive on their own, i.e $V(N)>\sum_{n \in N} V(\{n\})$.

Taking the previous modeling decisions into account, and considering economically sensible alliances the Nash bargaining solution is defined as the solution to Problem 2 ,

\section{Problem 2}

$$
\begin{aligned}
\max & \prod_{n \in N}\left(x_{n}^{n b s}-V(\{n\})\right)^{\alpha_{n}} \\
\text { s.t. } & \sum_{n \in N} x_{n}^{n b s}=V(N) \\
& x_{n}^{n b s} \geq V(\{n\}), \forall n \in N
\end{aligned}
$$

which can be proven to be given by the Equation (9).

$$
x_{n}^{n b s}=\frac{\alpha_{n}}{\sum_{j \in N} \alpha_{j}}\left(V(N)-\sum_{j \in N} V(\{j\})\right)+V(\{n\}) .
$$

Please note that when considering the contribution $\left(v_{n}\right)$ as bargaining power and for the case where the stand alone revenue is equal to zero $(V(\{n\}=$ $0, \forall n \in N)$, the Nash bargaining solution is equal to the Proportional share, defined in Sub-section 4.2. When modeling the bargaining power through stock $\left(c_{n}\right)$, and when the stand alone revenue of all members is equal to zero, the Nash bargaining solution splits the revenue proportional to the stock.

The Nash bargaining solution verifies interesting properties. Indeed, let $\Phi^{n b s}(\tau, \Delta)=\left\{\Phi_{n}^{n b s}(\tau, \Delta)\right\}_{n \in N}$ be the outcome determined by the Nash bargaining solution with set of possible outcomes $\tau$ and set of disagreement points $\Delta$. Nash proved that for 2-person bargaining games the following four axioms characterize the Nash bargaining solution [13, theory that was then extended to multiple players (see e.g. [16]).

- Symmetry: if $\tau$ is symmetric, and if players are indistinguishable, then players get the same outcome.

- Independence of irrelevant alternatives: let $\tau$ and $\tau^{\prime}$ be such that $\tau^{\prime} \subseteq \tau$. If $\Phi^{n b s}(\tau, \Delta) \in \tau^{\prime}$ then $\Phi^{n b s}\left(\tau^{\prime}, \Delta\right)=\Phi^{n b s}(\tau, \Delta)$

- Pareto efficiency: $\Phi^{n b s}(\tau, \Delta)$ is Pareto efficient if there is not $t \in \tau$ such that $t \geq \Phi^{n b s}(\tau, \Delta)$ and $t_{n}>\Phi_{n}^{n b s}(\tau, \Delta)$ for some $n \in N$. 
- Invariance to equivalent utility representations: a transformation of the utility functions that maintains the same ordering over preferences (e.g. a linear transformation), should not change the outcome of a bargaining process.

In addition, when modeling each member $n$ 's bargaining power by its stock $c_{n}$, monotonicity is verified for an economically sensible alliance, as we show in Theorem 2. For convenience, we shall from know on admit the notation $\Phi^{n b s}(N, c, U)$ to refer to the Nash bargaining solution applied to our context, as well as the short notation $x^{n b s}$.

Theorem 2 Incentive for improving stock of the Nash bargaining solution. Let the revenue maximizing alliance $(N, U, c)$ denote a bargaining problem where: the space of solutions is given by $X=\left\{\left(x_{1} \ldots x_{|N|}\right): \sum_{n \in N} x_{n}=V(N), x_{n} \geq\right.$ $V(\{n\}) \forall n \in N\}, V$ is the revenue function defined by Problem 1, the set of disagreement points is given by $\Delta=\{V(\{n\}), \forall n \in N\}$ and the bargaining powers are given by the stocks $c$. Let the alliance be economically sensible, that is to say $V(N)>\sum_{n \in N} V(\{n\})$. If $n \in N$ increases its stock, then its revenue share (i.e. $\Phi_{n}^{n b s}$ ) will be not decreased. That is, letting $\hat{c}$ represent the stock vector where $n$ 's stock is increased, $\Phi_{n}^{n b s}(N, \hat{c}, U) \geq \Phi_{n}^{n b s}(N, c, U)$, where $\Phi_{n}^{n b s}(N, c, U)$ is the Nash bargaining solution for member $n$.

Proof Without loss of generality we assume that the only member increasing its stock is $n \in N$. The share of $n$ through the Nash bargaining solution is given by:

$$
x_{n}^{n b s}=\frac{c_{n}}{\sum_{j \in N} c_{j}}\left(V(N)-\sum_{j \in N} V(\{j\})\right)+V(\{n\})
$$

we want to prove that $\frac{\partial x_{n}^{n b s}}{\partial c_{n}} \geq 0$.

$$
\begin{aligned}
& \frac{\partial x_{n}^{n b s}}{\partial c_{n}}=\frac{\sum_{j \in N} c_{j}-c_{n}}{\left(\sum_{j \in N} c_{j}\right)^{2}}\left(V(N)-\sum_{j \in N} V(\{j\})\right) \\
& +\frac{\partial V(\{n\})}{\partial c_{n}}\left(1-\frac{c_{n}}{\sum_{j \in N} c_{j}}\right)+\frac{\partial V(N)}{\partial c_{n}}\left(\frac{c_{n}}{\sum_{j \in N} c_{j}}\right),
\end{aligned}
$$

which is easy to see has all terms equal to or greater than zero. Indeed, function $V$ is defined after a maximization problem -Problem 1 with non decreasing objective function. Increasing $c_{n}$ implies relaxing one of that problem's linear constraints, thus, increasing the functional value of $V$. Hence, $\frac{\partial V(N)}{\partial c_{n}}$ is positive or zero and analogously for $\frac{\partial V(\{n\})}{\partial c_{n}}$. In addition, since by hypothesis the alliance makes economic sense, the alliance's revenue is at least as large as the sum of all revenues each member would perceive on their own, i.e. $V(N) \geq \sum_{j \in N} V(\{j\})$ 


\begin{tabular}{|c|c|c|}
\hline \multirow{2}{*}{ Method } & \multicolumn{2}{|l|}{ Discussed properties } \\
\hline & Fulfilled & Not Fulfilled \\
\hline Shapley value & $\begin{array}{c}\text { Dummy player, Symmetry } \\
\text { Additivity, Monotonicity, Efficiency } \\
\text { Balanced Contributions }\end{array}$ & $\begin{array}{c}\text { Stability } \\
\text { No free riders }\end{array}$ \\
\hline Proportional & $\begin{array}{c}\text { Efficiency } \\
\text { Fairness }\end{array}$ & Stability \\
\hline Aumann-Shapley & Efficiency & Monotonicity \\
\hline Friedman-Moulin & Not applicable & Not applicable \\
\hline $\begin{array}{l}\text { Nash Bargaining } \\
\text { Solution }\end{array}$ & $\begin{array}{l}\text { Symmetry, Monotonicity, Pareto efficiency } \\
\text { Independence of irrelevant alternatives } \\
\text { Invariance to equivalent representations }\end{array}$ & $\begin{array}{l}\text { Stability } \\
\text { Fairness }\end{array}$ \\
\hline
\end{tabular}

Table 4: Summary of discussed properties for existing methods

In spite of the good properties of the Nash bargaining solution, modeling the bargaining power after the member's resources can provide with quite unfair results. For instance, consider an alliance where the stand alone revenue of all members are equal to zero, and where the contribution of the member with the greatest amount of resources is equal to zero. The Nash bargaining solution would give the greatest share to the member contributing the less. In addition, the Nash Bargaining solution applied to our problem can provide with unstable solutions, as we shall see through simulations in Section 6

As a conclusion to this section, Table 4 summarizes the different existing revenue sharing methods we have introduced, and the discussed properties regarding each of them.

\section{The Proposed Revenue Sharing Method}

Having seen that existing techniques are not suitable for our problem, we shall now propose a new method to perform the revenue sharing in the specific scenario of revenue functions given by utility maximization problems. We seek for a method fulfilling stability, efficiency, fairness and monotonicity. We first study the set of feasible solutions, defined as those who are stable and efficient. Subsequently, we focus on how to choose a point belonging to that set. Finally, we evaluate the different ways of choosing a point regarding fairness and monotonicity.

\subsection{The Feasible Solutions Set}

In order to have stability in the coalitions Inequalities (3) must hold, that is $\sum_{n: n \in \mathcal{Q}} x_{n} \geq V(\mathcal{Q}), \forall \mathcal{Q} \subseteq N$. Let us enumerate all the possible sub-coalitions $\mathcal{Q} \in N$ and index them using index $j=1 \ldots 2^{|N|}$. We rewrite Inequality (3) as a linear system as:

$$
Q x \geq \hat{v},
$$


where $Q=\left\{Q_{j, n}\right\}$ is a $2^{|N|} \times|N|$ matrix that indicates which members belong to each sub-coalition (i.e. $Q_{j, n}=1$ if member $n$ belongs to sub-coalition $j$ and 0 otherwise) and $\hat{v}=\left\{V\left(\mathcal{Q}_{j}\right)\right\}_{j=1 \ldots 2^{|N|}}$ is the vector that indicates in the $j$-th component the revenue of sub-coalition $j$.

We must consider at the same time the efficiency property, which we write as the vector representation of Equation (2):

$$
e^{T} x=V(N) .
$$

We refer to the set of points verifying Equation (12) and Equation (13) as to the feasible set. Depending on the alliance resources and the utility functions, the feasible set might determine a unique point, a non empty set included in $\mathbb{R}^{|N|}$, or an empty set. The following examples show two cases where the two latter situations occur.

Example, an empty feasible set Consider Topology C shown in Fig. 1c. The three services illustrated in the figure are offered. Nodes' capacities and utility functions are such that the revenue of the sub-coalitions are $V(N)=$ $V(\{1,2\})=5, V(\{2,3\})=4$ and $V(\{1,3\})=2$. In order to achieve stability the total revenue (5 monetary units) must be split in such a way that every route receives at least what they would receive alone. It is not difficult to see that this is not possible at the same time for all routes, since the following inequalities must hold: $x_{1}+x_{2} \geq 5, x_{1}+x_{3} \geq 2, x_{2}+x_{3} \geq 4$ and $x_{1}+x_{2}+x_{3}=5$. Hence, the feasible set is empty.

It is interesting to remark that for different values of the utility functions, and the same topology, the feasible set could be non-empty.

Example, a feasible region Consider now Topology A shown in Fig. 1a, Utility functions and node capacities are such that $V(N)=V(\{2,3\})=5$ and $V(\{1,3\})=2$. A feasible solution must fulfill $x_{1}+x_{3} \geq 2, x_{2}+x_{3} \geq 5$ and $x_{1}+x_{2}+x_{3}=5$. The vectors $x$ that satisfy all equations are $\{x=(0,3-\epsilon, 2+\epsilon)$ : $\epsilon \in \mathbb{R}, 0 \leq \epsilon \leq 3\}$, which corresponds to a segment in $\mathbb{R}^{2}$.

\subsection{The Choice of a Point within the Feasible Solutions Set}

For configurations with no solution, i.e. with empty feasible region, we claim that the coalition for those utility functions should not exist as such, since there is no revenue sharing method that can make it stable. Therefore, we focus our attention on the case where constraints (12) and (13) determine a non-empty region. In order to choose a point from such region we formulate the following Optimization Problem:

\section{Problem 3}

$$
\begin{aligned}
& \quad \min _{x} f(x) \\
& \text { s.t. } Q x \geq \hat{v}, e^{T} x=V(N),
\end{aligned}
$$


where $f(x)$ is a convex function. Please note that we can dispense with the restriction of non negative revenue shares, since it is already considered by the stability property. Indeed, the constraints $Q x \geq \hat{v}$ include constraints of the form $x_{n} \geq V(\{n\}), \forall n \in N$ and by definition $V(\{n\})$ is non negative.

Problem 3 constitutes a family of methods which can be tuned to cover additional properties by considering different objective functions. We shall explore the additional properties they provide - or not provide- in the following subsection, through simulations. Let us first introduce different objective functions.

\subsubsection{Projections}

One possible natural approach is to consider either the Shapley value, The Nash bargaining solution or the Proportional share and project either of these vectors onto the feasible set. The method would inherit the good properties of the Shapley value, the Nash bargaining solution or the Proportional share as appropriate, when the share is already in the feasible set, and otherwise it would return the closest value. In those cases the objective functions of Problem 3 would be $f(x)=\left\|x-x^{s h}\right\|^{2}, f(x)=\left\|x-x^{n b s}\right\|^{2}$ or $f(x)=$ $\left\|x-x^{p r}\right\|^{2}$, where the square of the norm is considered in order to have a quadratic programming optimization problem.

Another possibility is to project the contributions vector $v$ onto the feasible set. Intuitively this would behave well as a sharing rule, since we are choosing the point the closest to the contributions vector. Please note that the proportional share is actually a linear transformation of the contributions vector. However, the projection onto a polytope is not a linear transformation so the results of projecting contributions vector $v$ and the Proportional share need not to be the same. In this case, the objective function takes the form $f(x)=\|x-v\|^{2}$.

\subsubsection{Equalization of the shares}

Yet another candidate to the objective function is the square of the Euclidean norm of the revenue share vector, that is $f(x)=\|x\|^{2}$. This objective function would intuitively provide with more even shares among members. On the other hand, this function does not keep any record of members' contributions to the revenue, thus monotonicity and fairness are likely not to be fulfilled.

\subsubsection{Weighted sums}

Another intuitive candidate for the objective function is one that shares proportional to either the stock or the contributions vector. We shall consider thus the sum of the shares weighted by either, the stock or the contribution of each node. This comes to a linear objective function and in order to have a convex function we consider the opposite of the weighted sum, that is $f(x)=-c^{T} x$ 
or $f(x)=-v^{T} x$. Intuitively this criterion would give more share to highest weights.

\section{Numerical Evaluation}

Simulations were performed with two different objectives, namely to further show the need of a new revenue sharing method for revenue maximizing alliances, and to evaluate the proposed method. The simulations presented in this work were performed on a regular computer with an intel i7 prof $3.5 \mathrm{GHz}$ processor and 8 GB of RAM memory running Debian Jessie. The optimization problems were solved using solver MINOS through AMPL. In all simulations we shall consider logarithmic utility functions.

Throughout the following subsections we shall illustrate the problem studied and the proposed solution with the concrete application of the alliance of NSPs, introduced in Section 2.1. This application is a particular case of a revenue maximizing alliance. Counterexamples in that setting prove that some methods are not appropriate for revenue maximizing alliances. Moreover, we evaluate the behavior of our method through extensive simulations, which constitute great evidence of the good behavior of the proposed solution.

\subsection{The need of a new revenue sharing method}

In this section we compute the revenue share for different topologies, using the Shapley value, defined by Equation (4), the proportional share, defined by Equation (5) and the Nash bargaining solution (Equation (9)). We then compare the results against the projection of those values into the feasible set. In order to calculate the projection, Problem 3 is solved setting $f(x)=$ $\left\|x-x^{s h}\right\|^{2}, f(x)=\left\|x-x^{p r}\right\|^{2}$ and $f(x)=\left\|x-x^{n b s}\right\|^{2}$.

Consider Topology D, shown in Fig. 2a, Consider that all nodes have capacity equal to 1 , and the utility function for service $s$ is of the form $U_{s}\left(a_{s}\right)=$ $\alpha_{s} \cdot \log \left(1+\beta_{s} \cdot a_{s}\right)$. The values of the parameters for this example, along with the solution of the revenue maximizing allocation problem (Problem 1), are show in Table 2b, Revenue sharing using the existing methods and their projection into the feasible set are shown in Table $2 \mathrm{c}$ (where notation $x_{\perp}^{*}$ stands for the projection of $*$ into the feasible set), along with the value of the contribution $v_{n}$ of each node.

Results in Table 2c show that the Shapley value and the Nash bargaining solution with nodes capacity as bargaining power do not lie in the feasible set. We can easily verify this by noting that projections are different to the original vectors. Consequently, as aforementioned, such methods are not suitable for our problem. Please note that since the stand alone revenue of all nodes in the topology is zero, the Nash bargaining solution with nodes contribution as bargaining power $\left(x^{n b s_{v}}\right)$ coincides with the Proportional share. 


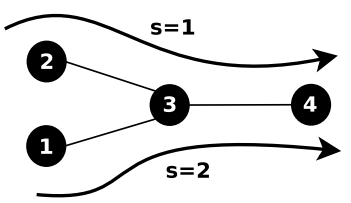

(a) Topology D

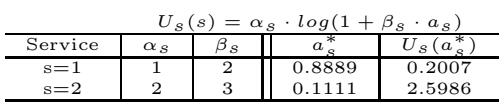

(b) Utility functions, and solution of the revenue maximizing problem.

\begin{tabular}{|c|c|c|c|c|c|c|c|}
\hline \multicolumn{7}{|c|}{ Revenue Share (\%) } & \multirow{2}{*}{${ }_{n}$} \\
\hline$x_{\perp}^{s v}$ & $x^{s v}$ & $x_{\perp}^{p r}$ & $x^{p r}$ & $x^{n b s_{v}}$ & $x_{\perp}^{n b s_{c}}$ & $x^{n b s_{c}}$ & $v_{n}$ \\
\hline 0 & 0.173 & 0 & 0 & 0 & 0 & 2.426 & 0 \\
2.773 & 2.715 & 2.737 & 2.737 & 2.737 & 3.235 & 2.426 & 7.625 \\
3.466 & 3.408 & 3.4834 & 3.484 & 3.484 & 3.235 & 2.426 & 9.704 \\
3.466 & 3.408 & 3.4834 & 3.484 & 3.4834 & 3.235 & 2.426 & 9.704 \\
\hline
\end{tabular}

(c) Revenue sharing using existing methods, and their projection into the feasible set. Shapley Value and Nash bargaining solution may not lie in the feasible region for such a topology.
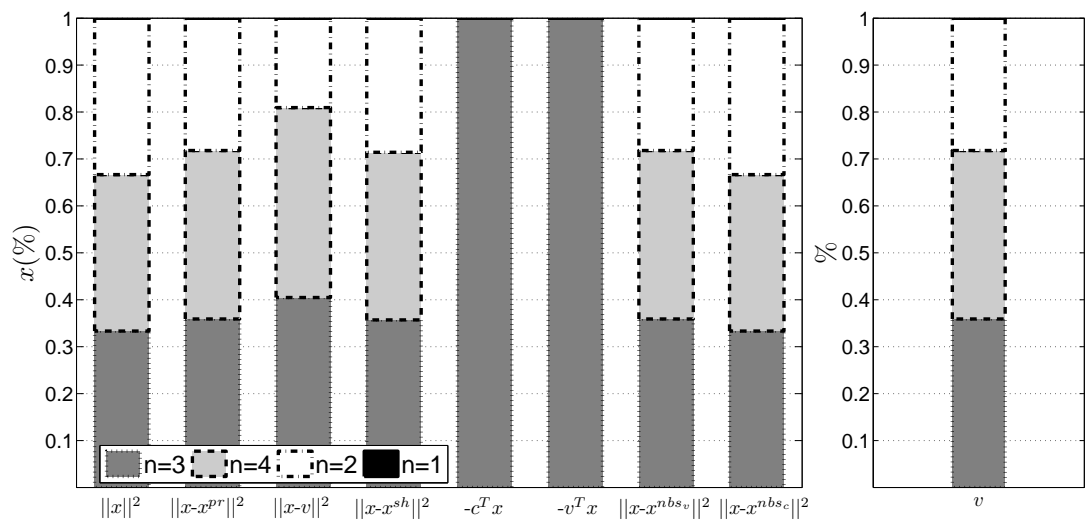

(d) Revenue sharing using the proposed method and different objective functions. Linear objectives functions are not fair; they might not respect the equal treatment of equals property.

Fig. 2: NSPs Alliance Example. Illustration of the need of a new sharing method and evaluation of the proposed method.

We now turn our attention to Topology E shown in Fig. 3a, Capacities are equal to one unit, utility functions and the solution to the allocation problem are show in Table 3b, Revenue shares using the different existing methods and their projection into the feasible set are shown in Table 3c. Results show that the Proportional share and Nash bargaining solutions do not lie to the feasible set. Such methods are thus not suitable in our case. 


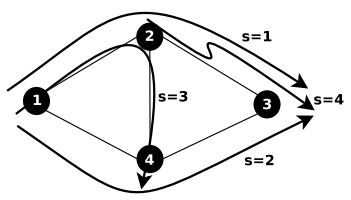

(a) Topology E

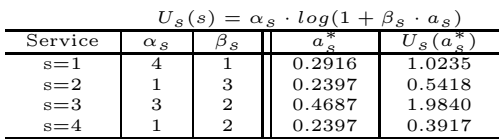

(b) Utility functions, and solution of the revenue maximizing problem.

\begin{tabular}{|c|c|c|c|c|c|c|c|}
\hline \multicolumn{7}{|c|}{ Revenue Share (\%) } & \multirow{2}{*}{} \\
\cline { 1 - 7 }$x_{\perp}^{s v}$ & $x^{s v}$ & $x_{\perp}^{p r}$ & $x^{p r}$ & $x^{n b s v}$ & $x_{\perp}^{n b s_{c}}$ & $x^{n b s_{c}}$ & $v_{n}$ \\
\hline 1.207 & 1.207 & 1.374 & 1.464 & 1.464 & 1.374 & 1.511 & 2.749 \\
2.162 & 2.162 & 2.202 & 2.112 & 2.112 & 1.648 & 1.511 & 3.965 \\
1.469 & 1.469 & 1.094 & 1.004 & 1.004 & 1.648 & 1.511 & 1.886 \\
1.207 & 1.207 & 1.374 & 1.464 & 1.464 & 1.374 & 1.511 & 2.749 \\
\hline
\end{tabular}

(c) Revenue sharing using existing methods, and their projection into the feasible set. Proportional share and Nash bargaining solution may not lie in the feasible region for such a topology.

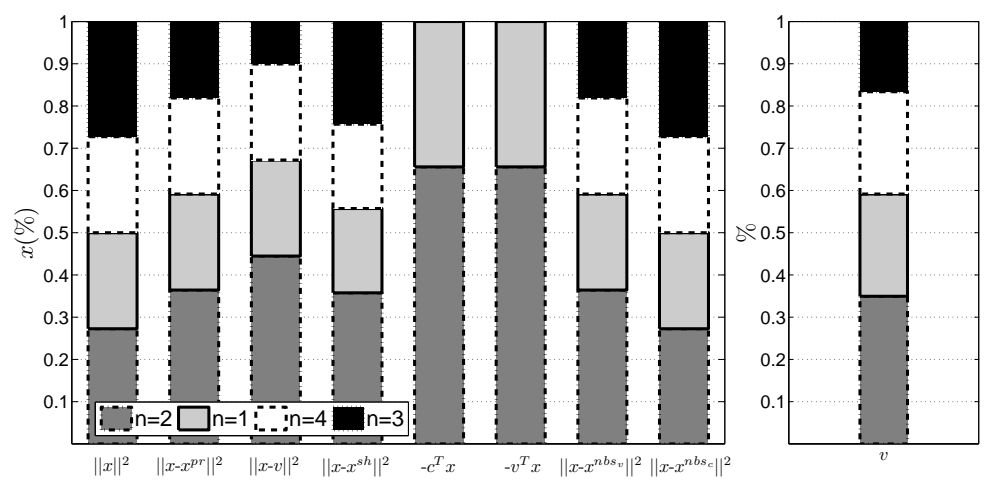

(d) Revenue sharing the proposed method and different objective functions. Linear objectives functions are not fair; they might not respect the equal treatment of equals property.

Fig. 3: NSPs Alliance Example. Illustration of the need of a new sharing method and evaluation of the proposed method.

Finally, consider Topology F, shown in Fig. 4a, utility functions and the solution to Problem 1 are shown in Table 4b Results in Table 4c show that Nash bargaining solution with capacity as bargaining power, and the Shapley value do not lie into the feasible region. Once again, this can be verified by comparing the values with their projection. In addition, those methods do not verify the no free riders property, as node 7 has a null contribution to the coalition $\left(v_{7}=0\right)$, while its revenue share according to the Shapley value is greater than zero $\left(x_{7}^{s v}=0.093\right)$ and the same occurs with the Nash bargaining 
solution $\left(x^{n b s_{c}}=4.268\right)$. So, once again, those solutions are not suitable to our problem.

\subsection{Behavior of the Proposed Revenue Sharing Method}

We now evaluate the proposed method through simulations, and explore the impact of using the different objective functions introduced in Subsection 5.2 , By construction, all solutions verify the efficiency and stability properties. Hence, we are interested in evaluating their behavior regarding fairness and monotonicity. We shall thus divide the simulation studies into those two cases, as presented in the following. Subsequently, we provide a summary of the simulation studies.

\subsubsection{Fairness evaluation}

We consider again the example in Fig. 2. Results using the proposed method with the different objective functions are shown in Fig. 2d.

Fig. $2 \mathrm{~d}$ should be read as follows. On the right-hand side of the figure nodes' contributions are shown as reference values to ease the interpretation of the results. These values are normalized, thus the Proportional share coincides with the contributions vector. Nodes' contributions are stacked up in descending order, bottom-up from the highest contribution to the lowest. This same order is respected in all bars and in the legend. On the left-hand side of the figure each bar corresponds to the stacked shares computed with the proposed method and with different objective functions. Shares are as well stacked up following the order imposed by the contributions.

Fig. 2d shows that considering the weighted sums as objective functions does not provide fairness. Indeed, the equal treatment of equals property is not verified. We can readily see that, while the contributions of nodes 3 and 4 are the same, their shares differ significantly since node 3 is getting all the revenue and node 4 receives no revenue. Moreover, with a linear objective function Problem 3 does not necessarily have a unique solution, which is the case in this example. Indeed, since the criterion is linear, it can be proved that the minimum is found at a vertex of the feasible set. Any vertex minimizing $-\sum\left(x_{1}+x_{2}+x_{3}+x_{4}\right)$ or $-\sum\left(x_{1} \cdot v_{1}+x_{2} \cdot v_{2}+x_{3} \cdot v_{3}+x . v_{4}\right)$ for the cases weighted by the capacities and by the contributions respectively, is a solution to the revenue sharing problem (i.e. a solution to Problem 3). We discard the use of linear functions since they neither provide with a unique solution nor they provide fairness.

Consider Topology E, shown in Fig. 3d, and objective function $f(x)=$ $\|x\|^{2}$. While, for instance, node 1's contribution is greater than node 4's contribution, they all receive the same share. Order preserving is then verified according to its definition (see Table 2) in a loose sense, that is accepting If $v_{n}>v_{j}$ then $x_{n}=x_{j}$. In addition, as commented above, considering this objective function tends to equalize the shares. 
Results for Topology $\mathrm{F}$ are shown in Fig. $4 \mathrm{~d}$, and show that the no free riders property is verified by all the considered functions.

In addition, results with all three topologies show that projecting the Shapley value, the Proportional share, the contributions vector and the Nash bargaining solutions, behave well with respect to our definition of fairness. In all cases order preserving, no free riding and equal treatment of equals are fulfilled.

As a consequence of the previous simulations we discard the use of weighted sums as objective functions, since as shown above they provide neither a unique solution nor fairness. Following simulations focus on the objective functions that involve the projection of the Shapley value, the Proportional share, the Nash bargaining solutions or the contributions vector.

\subsubsection{Monotonicity Evaluation}

We consider Topology A, where nodes capacities are equal to one unit and utility functions are those shown in Table 5a Fig. $5 \mathrm{~b}$ shows the revenue share of node 3 computed using the proposed method, as a function of the capacity of node 3 .

This simple example shows that $f(x)=\left\|x-x^{p r}\right\|^{2}, f(x)=\left\|x-x^{s h}\right\|^{2}$, $f(x)=\|x\|^{2}, f(x)=\left\|x-x^{n b s_{v}}\right\|^{2}$ and $f(x)=\left\|x-x^{n b s_{c}}\right\|^{2}$ do not provide monotonicity. Indeed, as long as node 3 is the bottleneck of both services, its share increases when its capacity increases. Once node 3 is no longer the bottleneck, the increase in its capacity produces, of course, an increase in the total revenue, but however a decrease in its own share for all the objective functions but $f(x)=\|x-v\|^{2}$. The revenue shares for all nodes according to the proposed method with $f(x)=\|x-v\|^{2}$ are shown in Fig. [5c, Just below, in Fig. $5 \mathrm{dd}$, the contribution vector for the different values of node 3's capacity is shown. These two figures allow as to check that fairness is as well verified.

\subsection{The best-behaved objective function}

In order to further evaluate the usage of the projection of the contribution vector as objective function, network topologies were generated using the automatic Internet topology generator BRITE 1], which automatically generates a graph with directed links and randomly assigns capacities, and receives as input the number of nodes and certain parameters for the algorithms running inside. Two of the evaluated network topologies are shown in Fig. 6a and Fig. 6b. Over each topology, we have defined an alliance where services are defined between every couple of nodes, provided a path in the topology between those nodes exists. For those services, the second shortest path between ingress and egress node has been chosen. For each service, we have defined utility functions of the form $U_{s}\left(a_{s}\right)=\alpha_{s} \log \left(1+\beta_{s} a_{s}\right)$. The parameters defining the alliance are shown in Table 6c. 


\begin{tabular}{|c|c|c|c|c|c|c|}
\hline \multirow[b]{2}{*}{ Property } & \multicolumn{6}{|c|}{ Objective function $f(x)$} \\
\hline & $\|x\|^{2}$ & $\left\|x-x^{s h}\right\|^{2}$ & $\left\|x-x^{p r}\right\|^{2}$ & $\|x-v\|^{2}$ & $\begin{array}{l}-v^{T} x \\
-c^{T} x\end{array}$ & $\begin{array}{l}\left\|x-x^{n b s_{v}}\right\|^{2} \\
\left\|x-x^{n b s_{c}}\right\|^{2}\end{array}$ \\
\hline Stability & $\checkmark$ & $\sqrt{ }$ & $\checkmark$ & $\checkmark$ & $\checkmark$ & $\checkmark$ \\
\hline Efficiency & $\checkmark$ & $\checkmark$ & $\checkmark$ & $\checkmark$ & $\checkmark$ & $\checkmark$ \\
\hline Eq. treat. of eq. & $x$ & $\check{\sim}$ & $\check{\sim}$ & $\check{\sim}$ & $x$ & $\check{\sim}$ \\
\hline Order preserving & $x$ & $\check{\sim}$ & $\check{\sim}$ & $\approx$ & $x$ & 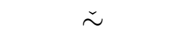 \\
\hline No free riders & $\check{\sim}$ & $\check{\sim}$ & $\check{\sim}$ & $\check{\sim}$ & $\check{\sim}$ & $\check{\sim}$ \\
\hline Monotonicity & $x$ & $x$ & $x$ & $\check{\sim}$ & 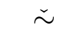 & $x$ \\
\hline
\end{tabular}

Table 5: Summary of the properties provided by the proposed method according to the objective function. $(\checkmark)$ fulfillment, $(\times)$ no fulfillment, $(\tilde{\sim})$ no counter example found. The projection of the contributions vector fulfills all desirable properties.

For each alliance, we have built a number of 100 different scenarios, by randomly sampling parameters $\alpha_{s}$ and $\beta_{s}$, for each service $s$. For each scenario, nodes capacities were varied one node at a time, from a value of $c_{n}=0$ till $50 \%$ more of their nominal capacity. With each value of capacity, revenue sharing was computed using the proposed method. Monotonicity was verified for all the evaluated scenarios, along all the evaluated values of capacities. In addition, fairness (i.e. order preserving accepting the loose definitions, no free riders and equal treatment of equals) was verified along all the tested scenarios and values of $c$. For Topology $\mathrm{G}$ and $\mathrm{H}$, the mean computation time was 0.09 and 0.18 seconds, respectively.

All in all, we have shown that the objective function of the proposed revenue sharing problem, that is of (Problem 3), has a great influence in the properties fulfilled by the revenue shares obtained by the proposed method. Table 5 summarizes the evaluated functions and the obtained results.

Results allow to conclude that the projection of the contributions vector, that is considering $f(x)=\|x-v\|^{2}$, presents the desirable properties. Indeed, stability and efficiency are verified by construction, and monotonicity and fairness were verified in all simulations. Please note that the exhibited properties were verified by the proposed method in all of the several scenarios tested by the authors, further than those showed in this paper. Interested readers can refer to 3 for further simulation results.

\section{Implementation Considerations}

Two distinct steps are part of our approach. A first step is resource allocation. We shall refer to this stage as to the selling phase. Resource allocation can be solved in a distributed, iterative manner, exactly as has been proposed by Kelly [10. That is, with a dual-based distributed algorithm, where Lagrangian multipliers and resources allocated are iteratively updated, and where each buyer and the network solve, at each iteration, a local utility maximization 
problem. The second step, once the revenue of the alliance is calculated, is to compute the revenue shares. We shall refer to this step as to the revenue sharing phase.

Our proposed method for revenue sharing necessitates having a centralized entity, trusted by the alliance, in order to compute the shares. This centralized entity must be aware of the resources provided by each member of the alliance, the services that are offered, and the utilities of each of these services. With this information, the centralized entity is able to compute all the information necessary for solving Problem 3, In particular, this implies solving Problem 1 for all possible subcoalitions.

In order to alleviate computation complexity, the centralized entity can, instead of computing the revenue share immediately after each selling phase, compute the revenue share after a given number of selling phases, for instance after all the selling phases that occur over one month. In that case, the revenue to be shared corresponds to the cumulated revenue during that period. During the considered period, the resources are assumed to remain constant. In addition, statistics must be considered with respect to the utility functions. For instance, considering the mean utilities during that period. The reader is referred to our previous paper 4] for a deeper discussion about this approach.

\section{Conclusion}

We have addressed the problem of revenue sharing in the context of revenue maximizing alliances, where resources are allocated according to the Network Utility Maximization problem (NUM). We have illustrated the pertinence of such a revenue maximizing allocation rule in several environments. We have defined and discussed the desirable properties for a revenue sharing method for such an alliance. We have then presented a comprehensible review of existing revenue sharing methods, and discussed their properties. We have shown that none of them verifies at the same time the desirable properties, i.e. efficiency, stability, monotonicity and fairness.

As a consequence, we have proposed a new revenue sharing method for revenue maximizing alliances, based on projecting members' contributions to the alliance onto a feasible set, conceived for providing economic stability and efficiency. In addition, the proposed method has shown, through extensive simulation studies, to behave well with respect to fairness and monotonicity, i.e. to provide incentives to the members of the alliance to increase their stock of resources provided to the alliance.

In this work we have not considered the dynamics of alliance formation, which constitutes an interesting research line we would like to address in future work.

Acknowledgements This work was partially founded by CSIC groups grants, Uruguay and Stic Amsud PROVE project. 


\section{References}

1. BRITE: Boston Representative Internet Topology Generator [Online] Available: http://www.cs.bu.edu/brite

2. ETICS: Economics and Technologies for Inter-carrier Services. European FP7 Research Project [Online] Available: http://www.ict-etics.eu

3. Amigo, I.: Technological and Economic Aspects for Quality of Service in Multidomain Alliances. Ph.D. thesis (2013)

4. Amigo, I., Belzarena, P., Vaton, S.: On the problem of revenue sharing in multi-domain federations. In: Networking 2012, Prague, Czech Republic (2012)

5. Aumann, R.J., Shapley, L.S.: Values of non-atomic games. Princeton University Press (1974)

6. Bethanabhotla, D., Caire, G., Neely, M.J.: Joint transmission scheduling and congestion control for adaptive streaming in wireless device-to-device networks. In: Signals, Systems and Computers (ASILOMAR), 2012 Conference Record of the Forty Sixth Asilomar Conference on, pp. 1179-1183. IEEE (2012)

7. Bogomolnaia, A., Holzman, R., Moulin, H.: Sharing the Cost of a Capacity Network. Mathematics of Operations Research 35(1), 173-192 (2010)

8. Friedman, E., Moulin, H.: Three Methods to Share Joint Costs or Surplus. Journal of Economic Theory $\mathbf{8 7}(2)$, 275-312 (1999)

9. Hougaard, J.L.: An Introduction to Allocation Rules. Springer (2009)

10. Kelly, F.P., Maulloo, A.K., Tan, D.K.H.: Rate Control for Communication Networks: Shadow Prices, Proportional Fairness and Stability. The Journal of Operational Research Society 49(3), 237-252 (1998)

11. Moulin, H., Laigret, F.: Equal-need sharing of a network under connectivity constraints. Games and Economic Behavior 72(1), 314-320 (2011)

12. Myerson, R.B.: Conference structures and fair allocation rules. International Journal of Game Theory 9(3), 169-182 (1980)

13. Nash, J.F.J.: The Bargaining Problem. Econometrica 18(2), pp. 155-162 (1950)

14. Osborne, M.J., Rubinstein, A.: A Course in Game Theory, first edn. The MIT Press (1994)

15. Rubinstein, A.: Perfect Equilibrium in a Bargaining Model. Econometrica 50(1), pp. 97-109 (1982)

16. Rubinstein, A., Osborne, M.J.: Bargaining and Markets. Economic theory, econometrics, and mathematical economics series. Academic Press, San Diego (1990). Notes bibliogr

17. Samadi, P., Mohsenian-Rad, A.H., Schober, R., Wong, V., Jatskevich, J.: Optimal RealTime Pricing Algorithm Based on Utility Maximization for Smart Grid. In: First IEEE International Conference on Smart Grid Communications, pp. 415-420 (2010)

18. Shapley, L.: A value for n-person games. In: H. Kuhn, A. Tucker (eds.) Contributions to the Theory of Games, vol. 28, pp. 307-317 (1953)

19. Shoham, Y., Leyton-Brown, K.: Multiagent Systems: Algorithmic, Game-Theoretic, and Logical Foundations. Cambridge University Press, Cambridge, UK (2009)

20. Yi, Y., Chiang, M.: Stochastic network utility maximisation-a tribute to Kelly's paper published in this journal a decade ago. European Transactions on Telecommunications 19(4), 421-442 (2008)

21. Young, H.: Monotonic solutions of cooperative games. International Journal of Game Theory 14, 65-72 (1985) 


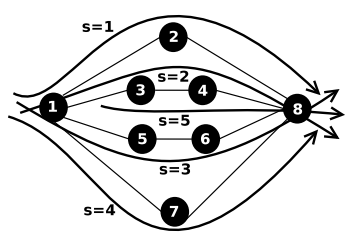

(a) Topology F

\begin{tabular}{c|c|c||c|c}
\multicolumn{5}{c}{$U_{S}(s)=\alpha_{S} \cdot \log \left(1+\beta_{S} \cdot a_{S}\right)$} \\
\hline Service & $\alpha_{S}$ & $\beta_{S}$ & $a_{S}^{*}$ & $U_{S}\left(a_{S}^{*}\right)$ \\
\hline $\mathrm{s}=1$ & 8 & 3 & 5.3922 & 22.7483 \\
$\mathrm{~s}=2$ & 2 & 2 & 0.9314 & 2.1036 \\
$\mathrm{~s}=3$ & 5 & 1 & 2.5784 & 6.3746 \\
$\mathrm{~s}=4$ & 1 & 1 & 0 & 0 \\
$\mathrm{~s}=5$ & 2 & 3 & 1.0980 & 2.9145 \\
\hline
\end{tabular}

(b) Utility functions, and solution of the revenue maximizing problem.

\begin{tabular}{|c|c|c|c|c|c|c|c|}
\hline \multicolumn{7}{|c|}{ Revenue Share (\%) } & \multirow{2}{*}{$v_{n}$} \\
\hline$x_{\perp}^{s v}$ & $x^{s v}$ & $x_{\perp}^{p r}$ & $x^{p r}$ & $x^{n b s_{v}}$ & $x_{\perp}^{n b s_{c}}$ & $x^{n b s_{c}}$ & \\
\hline 10.026 & 9.910 & 10.345 & 10.345 & 10.345 & 9.488 & 4.268 & 27.273 \\
6.853 & 6.738 & 6.540 & 6.540 & 6.540 & 9.488 & 4.268 & 17.243 \\
1.226 & 1.470 & 0.930 & 0.930 & 0.930 & 1.226 & 4.268 & 2.453 \\
1.226 & 1.470 & 0.930 & 0.930 & 0.930 & 1.226 & 4.2678 & 2.453 \\
1.246 & 1.131 & 1.223 & 1.223 & 1.223 & 1.612 & 4.268 & 3.224 \\
1.246 & 1.131 & 1.223 & 1.223 & 1.223 & 1.612 & 4.268 & 3.224 \\
0 & 0.093 & 0 & 0 & 0 & 0 & 4.268 & 0 \\
12.315 & 12.199 & 12.950 & 12.950 & 12.950 & 9.488 & 4.268 & 34.141 \\
\hline
\end{tabular}

(c) Revenue sharing using existing methods, and their projections into the feasible set. Nash bargaining solutions with capacity as bargaining power and Shapley value might not fulfil the no free riders property.
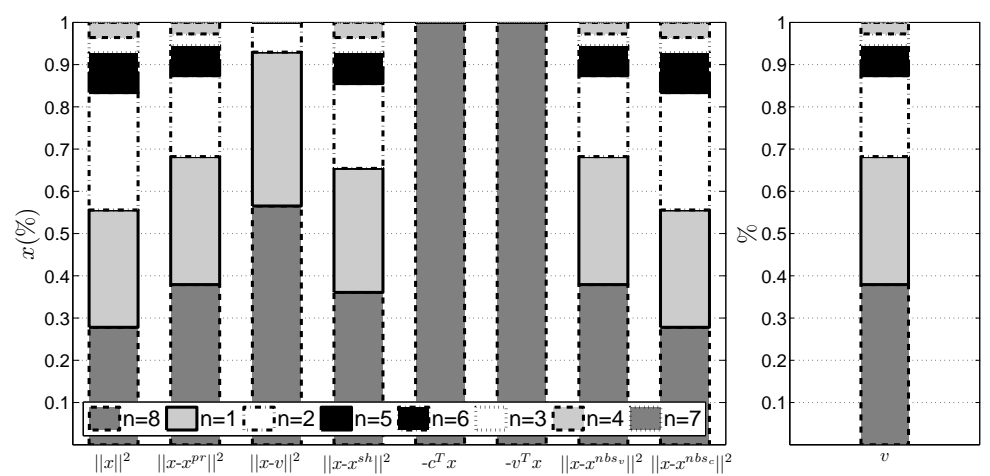

(d) Revenue sharing using the proposed method with different objective functions. Linear objectives functions are not fair; they might not respect the equal treatment of equals property.

Fig. 4: NSPs Alliance Example. Illustration of the need of a new sharing method and evaluation of the proposed method. 


\begin{tabular}{c|c|c}
\multicolumn{3}{c}{$U_{s}\left(a_{s}\right)=\alpha_{s} \cdot \log \left(1+\beta_{s} \cdot a_{s}\right)$} \\
\hline Service & $\alpha_{s}$ & $\beta_{s}$ \\
\hline $\mathrm{s}=1$ & 2 & 1.1 \\
$\mathrm{~s}=2$ & 1.5 & 2 \\
\hline
\end{tabular}

(a) Utility functions, and solution of the revenue maximizing problem for Topology A.

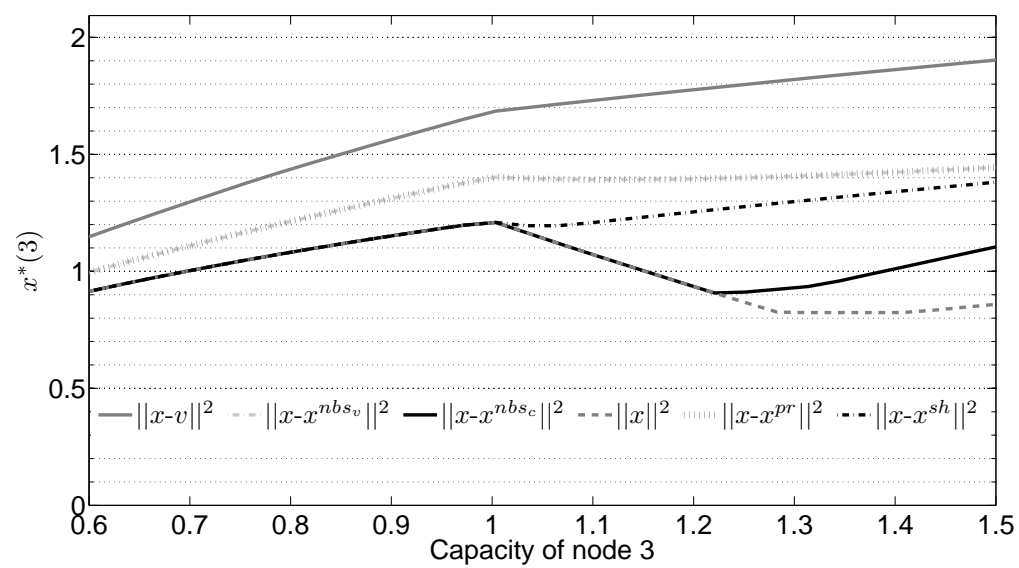

(b) Revenue share of node 3 using the proposed method with different objective functions for an increasing capacity of node 3 . Monotonicity is verified only when $f(x)=\|x-v\|^{2}$.

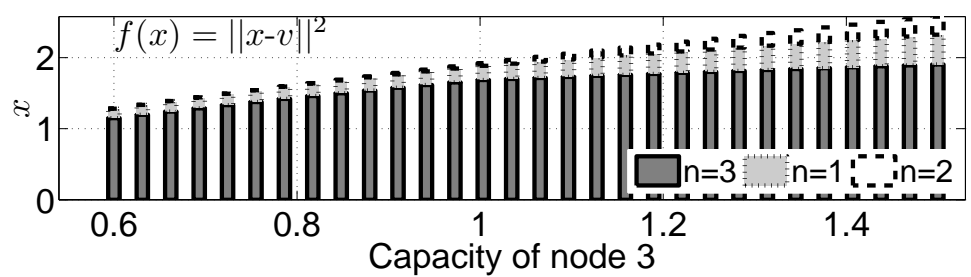

(c) Revenue share of all nodes using the proposed method with $f(x)=\|x-v\|^{2}$, as a function of node 3's capacity.

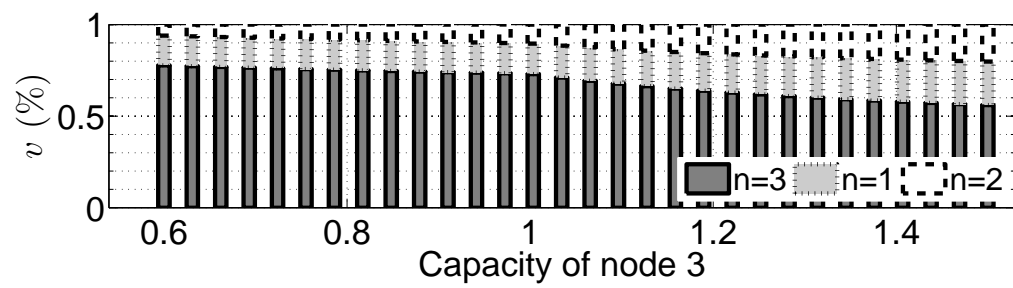

(d) Contribution vector as a function of node 3's capacity.

Fig. 5: Revenue sharing with the proposed method, results for Topology A. 


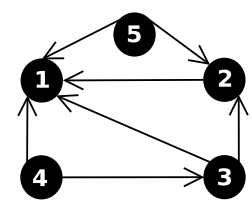

(a) Topology G.

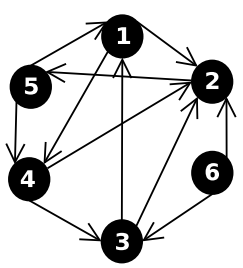

(b) Topology H.

\begin{tabular}{|c|c|c||c|}
\hline Topology & $\begin{array}{c}\text { Nominal } \\
\text { capacity }\end{array}$ & Services & $\begin{array}{c}\text { Utility functions } \\
U_{s}\left(a_{s}\right)=\alpha_{s} \log \left(1+\beta_{s} a_{s}\right)\end{array}$ \\
\hline \multirow{3}{*}{$\mathrm{G}$} & $\mathrm{c}=(340,426,200$ & $(3,2,1)$ & $\alpha_{s} \sim \exp (4)$ \\
& $522,365)$ & $(4,3,1)$ & $\beta_{s} \sim \exp (6)$ \\
& & $(5,2,1)$ & \\
& & $(5,4,4,2)$ & \\
& & $(3,1,2)$ & $\alpha_{s} \sim \exp (4)$ \\
& $\mathrm{c}=(718,586,866$ & $(4,3,2))$ & $\beta_{s} \sim \exp (6)$ \\
& $482,416,831)$ & $(5,4,2)$ & \\
& & $(6,3,2)$ & \\
& & $(5,1,4,3)$ & \\
& & $(5,1,4)$ & \\
\hline
\end{tabular}

(c) Parameters defining the tested alliances.

Fig. 6: Two of the automatically generated network topologies used on simulations. The proposed method with $f(x)=\|x-v\|^{2}$ verified monotonicity, and fairness. 